\title{
La historia oral y sus aportaciones a la investigación educativa
}

\author{
Romelia Hinojosa Luján \\ Profesora investigadora \\ Secretaría de Educación, Cultura y Deporte del Estado de Chihuahua
}

\section{Resumen}

$\gamma$ ste ensayo tiene el propósito de reflexionar sobre la historia oral como enfoque epistemológico y metodológico en la investigación social. En primera instancia se hace un análisis conceptual de los términos utilizados en el enfoque y se trabajan algunas recomendaciones metodológicas y técnicas. Por último se analiza la pertinencia de la historia oral en algunas temáticas de la investigación educativa y se plantean algunos retos que tendría que afrontar su aplicación en el campo educativo.

Palabras clave: historia oral, investigación biográfica, relatos de vida, historias de vida, entrevistas.

\section{Origen y conceptos}

La historia oral surge en los Estados Unidos en la década de los años cuarenta. Comienza como una técnica que recupera los testimonios de personajes destacados de la historia o testigos fundamentales y camina transformando lo que a su paso encuentra, hasta posicionarse como método de investigación en la historia, la antropología y la sociología.

La historia cultural, la microhistoria italiana, la historia de la vida cotidiana alemana, algunas corrientes feministas y posmodernas fueron los antecedentes de los Estudios Subalternos (Archila, 2005). Pero aún miembros de estas corrientes, pertenecien- 
tes al mundo universitario, despreciaban las fuentes orales por considerarlas poco rigurosas.

América Latina fue un campo fértil para la historia oral aunque en un primer momento se visualizó como complementaria de las fuentes escritas. Sin embargo esta visión luego se supera y se plantea no solamente como un enfoque técnico, sino como un posicionamiento epistemológico.

Una de las características de la Historia Oral es su naturaleza interdisciplinaria: se abreva de la antropología, de la sociología, de la teoría literaria y de las experiencias realizadas en la educación. La historia oral, se entiende como:

Un espacio de confluencia interdisciplinaria, que al surgir desde el seno de la historia social procede a seleccionar nuevos sujetos sociales, en escalas y niveles locales y regionales; con el fin de abordar fenómenos y cuerpos de evidencias específicas y controlables; con técnicas precisas y fuentes nuevas y plurales, que tiene el propósito de lograr aproximaciones cualitativas de los procesos y fenómenos sociales e individuales [...] por destacar y centrar su análisis en la visión y versión que desde dentro y lo más profundo de la experiencia, expresan los sujetos sociales considerados centralmente en el ámbito de la historia social-local-oral (Aceves, 1993: 234).

La historia oral tiene la particularidad de llenar el silencio y las ausencias de que adolece el material documental. Podemos encontrar, entre los usos de la historia oral, una utilidad a futuro y otra para el presente (Olivera, 1971); el primer caso alude al hecho de recopilar recuerdos, ideas y memorias de los informantes vivos, a través de la grabación, para que sean usados por quienes se dedican a historizar en el futuro; el segundo se refiere al uso inmediato del material recabado a través de las entrevistas a profundidad que lleven a producir historiografía. Sin embargo, su uso no se constriñe únicamente al campo de la historiografía educativa, puede también ser un recurso muy valioso para extenderse al campo de la investigación educativa.

El uso de la historia oral, no es privativo de la producción histórica. La antropología, la educación y la sociología (entre otras disciplinas), comparten sus usos y aportan a su crecimiento metodológico. Thompson (1993) aporta una reflexión muy rica en torno a la participación tan exitosa de la historia oral en el cambio de la sociología estructural hacia una visión que explique la participación del individuo en la construcción social de la realidad: "Tenemos que crear una sociología teóricamente más veraz y sustantivamente mejor consolidada, construyendo la teoría paso a paso, con descubrimiento de hechos; y el método de la historia de vida ofrece un instrumento vital para esa labor" (Thompson, 1993: 135). Equipara la historia oral con un enfoque crítico que provoca la intervención en la realidad, Portelli lo expresa de una manera sencilla: "el historiador oral ha producido el documento, ha influido en la realidad social en la que actúa" (Portelli, s/f: 87).

Es decir, a partir de testimonios o recomendaciones como la anterior, podemos decir que la historia oral es un canal vinculante que posibilita la interdisciplinariedad 
de las ciencias sociales y promueve la visión compleja de la realidad a la que Edgar Morin se refiere en su propuesta epistemológica (Morin y Pakman, 2003). Alessandro Portelli, también aporta algunas reflexiones sobre esta complejidad:

El tiempo es un continuo. Ubicar un acontecimiento en el tiempo requiere que se rompa el continuo y se lo vuelva discreto. Tal como ocurre con otros continuos -con los sonidos, por ejemplo- esta ruptura se produce en dos niveles: el de la sucesión lineal y el de la simultaneidad vertical. La periodización -el plano sintagmático- es el procedimiento con el que estamos más familiarizados: el tiempo se divide horizontalmente en periodos y en épocas, y se los "cuelga" de acontecimientos clave que funcionan como particiones e intérpretes de cada unidad secuencial (s/f:. 89).

Obsérvese cómo el plano filosófico de esta reflexión sobre la historia oral, nos remite a la relación entre el sujeto cognoscente y su objeto de conocimiento que realmente es un sujeto de conocimiento. Por ello se afirma que los planteamientos realizados para la historia oral rebasan el plano metodológico. Es necesario ahora describir algunos elementos que dibujan en el plano técnico.

Es difícil discurrir la frontera entre investigación biográfica y narrativa. Ambas son parte de la investigación cualitativa pero tienen enfoques particulares. Más compleja se torna la temática cuando inmiscuimos términos como historia oral, historias de vida, biografías, narrativas. Para autores como Bolívar y Domingo investigación biográfica es la investigación que se ocupa de todo tipo de fuentes que aportan información de tipo personal y que sirven para documentar una vida, una acontecimiento o una situación social, hace inteligible el lado personal y recóndito de la vida, de la experiencia, del conocimiento. En él tienen cabida todos los enfoques y vías de investigación cuya principal fuente de datos se extrae de biografías, material personal o fuentes orales, que dan sentido, explican o constan a preguntas vitales actuales, pasadas o futuras, a partir de la elaboración o posibles argumentos con los que se cuentan experiencias de vida o historias vividas desde la perspectiva de quien las narra [...] va más allá de una simple metodología para constituirse... en una perspectiva propia, situada en un espacio más amplio de investigación cualitativa en ciencias sociales, acorde con determinadas tendencias y posiciones de la sensibilidad postmoderna (2006: 12 y 110).

Los postulados básicos del enfoque biográfico son: la narración porque es difícil de percibir y transmitir de otro modo; es constructivista porque la historia se reconstruye, reinterpreta o re-focaliza; contextual puesto que los significados se interpretan y adquieren atendiendo al contexto histórico-social en el que se desenvuelven.

Generalmente se dice o se piensa que la ciencia busca la verdad. Un posicionamiento crítico nos permite comprender que toda verdad es relativa, entonces habrá que reformular algunas ideas en torno a la historia, sobre todo a la historia oral. Su búsqueda entonces se sujeta, no a la verdad, sino a lo que la memoria y el informante quieren manifestar sobre su recuerdo, por- 
que una parte del olvido es inconsciente y otra intencional. Doubrowsky afirma que cuando uno cuenta algo sobre sí, siempre cuenta cuentos (citado por Gaulejac y Silva, 2002).

Algunos de los conceptos y elementos imprescindibles de análisis de este tipo de investigación son: la memoria, la subjetividad, identidad y la construcción del sujeto.

La memoria es herramienta de la historicidad, confiere libertad, autonomía, creatividad. Nadie puede cambiar nuestra historia, pero sí nuestro recuerdo sobre ella y cómo éste actúa sobre nosotros en el presente. No es un simple registro del pasado en el que se acumulen los recuerdos. Es contradictoria pues se da la pugna del sujeto entre el ser y el querer ser.

La memoria de los sujetos es selectiva en algunos hechos; con esto mostramos que tienen una memoria y que el olvido es parte de ella: entonces, se dará a los sujetos la posición de testigos con plenos derechos, cuando sus opiniones sean sometidas a un análisis ajustado (Joutard, 1986).

No se puede hablar de memoria sin referirse a la identidad: la búsqueda del ¿quién soy? o ¿quiénes somos? Nos remite a la búsqueda en la memoria de nuestro pasado. De esta manera la memoria funda identidades: individual y colectiva (Gaulejac y Silva, 2002). He aquí uno de los principales campos propicios para la incursión de la historia oral y de la investigación biográfica en la educación: el estudio de las identidades.

\section{Técnicas e instrumentos de la historia oral}

La historia oral, dentro de este gran paradigma de producción biográfico-narrativa, se puede distinguir como un movimiento político que tiene por finalidad «dar voz a los sin voz».

La historia oral cuestiona fuertemente la objetividad que el positivismo enarbola. Pone en tela de juicio la tarea de la historia como forma explicativa de la realidad (Garay, 1999). La historia oral utiliza como métodos, técnicas e instrumentos: la historia de vida, la biografía narrativa, el relato de vida, la entrevista, etc.

Metodológicamente la historia oral abarca las siguientes fases: selección y determinación del tema y problema de investigación; conceptualización y construcción del entramado teórico; determinación de los objetivos y propósitos; revisión de la frontera del conocimiento en que se inscribe el trabajo; precisión de los pasos metodológicos; diseño y elaboración de los instrumentos de investigación; trabajo de campo mediante las entrevistas; sistematización y organización de las fuentes orales producidas; análisis del contenido; formulación de resultados y difusión de los hallazgos (Aceves, 1996).

La principal técnica de la historia oral es la entrevista, entendida como el recuento de la memoria de los hechos que al entrevistado vivió en el pasado, a través del cuestionamiento abierto y flexible que el entrevistador realiza para posibilitar el recuerdo. Para ello, es necesario que se realice una guía sumamente dúctil que sirva únicamente de pretexto para poder expresar el 
testimonio de vida de algún protagonista de un hecho histórico. Las preguntas se estructurarán, sin embargo, no serán rígidas y tendrán la flexibilidad suficiente para explotar vetas de información que proporcionen los entrevistados, sin que éstas hayan sido contempladas en las preguntas originales.

Para Thompson (1988) la entrevista es una relación social entre unas personas que tienen sus propias convenciones, y una violación de las mismas podría romperla. Fundamentalmente se espera que el entrevistador muestre interés por el informante, le permita expresarse sin constantes interrupciones, y le facilite algún tipo de guía sobre lo que se ha de tratar si es necesario... Una entrevista no es un diálogo ni conversación. Todo consiste en hacer que el informante hable. El entrevistador debe permanecer en un segundo plano tanto como sea posible (235).

La entrevista se traduce a textos que se convierten en unidades o fragmentos pero que al unirse conforman el relato de vida. El relato de vida es entonces el producto obtenido de la entrevista, también se le llama testimonio oral, que recupera lo que el entrevistado expresó a través de su testimonio motivado por las preguntas realizadas a través de un guión.

Existen muchas consideraciones técnicas sobre la entrevista: el rapport que se debe crear para propiciar un ambiente adecuado para expresar profundamente los recuerdos que vienen a la mente del entrevistado; el lugar; la necesidad y el permiso de grabar el testimonio; la ética en la publicación; la posibilidad de no agotar el relato histórico en una sola reunión, etc. (Querzoli, 2003).

Es importante apuntar que para la selección de los informantes puede privar el criterio de la representatividad o de la unicidad. Ambos son válidos y aportan elementos de interpretación para la realidad social.

Graciela de Garay (1999) nos proporciona una visión dialéctica de las entrevistas. Supera la tradicional definición de las preguntas "cara a cara», para proporcionarnos la idea de que la entrevista es un diálogo. Las grabaciones resultan una actividad conjunta negociada entre el entrevistado y entrevistador, se organizan a partir de las perspectivas históricas de ambos.

El entrevistador debe estar atento no solo a lo que se dijo, sino a cómo se dijo y quién lo dijo. Se entra entonces al territorio de la interpretación, por lo que se recomienda no guiar al entrevistado, no tener la intención de crear consensos, no interrumpirlo, ocultar nuestros puntos de vista, no interrumpir ni acosar con preguntas molestas. Debemos dejar que el texto narrativo fluya, porque también el orden, los olvidos, los traslapes, las omisiones, tienen significado.

Agréguese a estos planteamientos de riesgo y de reto, la posibilidad de la transcripción de la oralidad al texto. Son mundos muy diferentes: ¿cómo transcribir el llanto, la inseguridad o la felicidad? "La transcripción no debe verse como un traslado pasivo de signos, sino como parte decisiva de la «construcción» de los datos, o como una verdadera y completa traducción -es decir, como un momento genuinamente inter- 
pretativo" (Farías y Montero, 2005: 7). Es necesaria la imaginación y la creatividad en el proceso de análisis y obtención del dato. Esta es una visión contraria al positivismo (Roncaglia, 2004). El investigador se convierte en el principal medio de investigación.

Será motivo de otro texto más amplio, la recuperación de todos los cuidados técnicos que se deben tener en la historia oral: desde la planeación de la entrevista, hasta la transcripción y análisis de la misma. De manera sintética se enuncian algunos de los elementos que deben estar presentes antes, durante y después de la entrevista:

\section{Antes de la entrevista}

$\rightarrow$ Seleccionar informantes a través de criterios claros: muestra no generalizable, sino indicativa.

$\rightarrow$ Técnica de bola de nieve para hacerse de nombres de informantes.

$\rightarrow$ Contactar al entrevistado y ponerse de acuerdo con el mejor lugar para la entrevista (tomar en cuenta acústica, hora, espacio, tiempo, entre otras cosas).

$\rightarrow$ Manifestarle de manera clara y directa el objetivo de la entrevista.

$\rightarrow$ Elaborar el guión de preguntas generales.

$\rightarrow$ Preparar requerimientos técnicos: grabadora, baterías, libreta de apuntes, carta compromiso en caso de ser necesaria, etc.

\section{Durante la entrevista}

$\rightarrow$ Disponer de tiempo por si el informante desea tomar un café, ver fotografías, etc.

$\rightarrow$ Solicitar autorización para grabar.

$\rightarrow$ Garantizar anonimato o, en su caso la participación del entrevistado en la autoría.

$\rightarrow$ Iniciar con preguntas generales que ubiquen contextualmente al entrevistado.

$\rightarrow$ No leer las preguntas, sino elaborarlas de manera natural, buscando una organización lógica de las temáticas.

$\rightarrow$ Facilitar el ambiente para que el entrevistado se explaye de manera libre y natural.

$\rightarrow$ Mostrarse interesado a través del lenguaje corporal.

$\rightarrow$ Explorar nuevas vetas de información si la situación lo amerita.

$\rightarrow$ Tomar notas de las actitudes del entrevistado.

$\rightarrow$ Concluir la entrevista dejando abierta la posibilidad de un nuevo encuentro en caso de que sea necesario ampliar o complementar alguna información.

\section{Después de la entrevista}

$\rightarrow$ Hacer la transcripción lo más pronto posible.

$\rightarrow$ Quien realiza la entrevista debe transcribir el audio a texto.

$\rightarrow$ Realizar el análisis a través de matrices, software u otros recursos. 
$\rightarrow$ Escribir la historia de vida.

$\rightarrow$ Devolver la información al informante para que la revise (evaluar la conveniencia).

Por su naturaleza, la entrevista a profundidad se caracteriza por ser abierta y flexible: cada una de ellas tiene un toque distintivo que hace imposible cualquier posibilidad de replicarse.

Los relatos de vida se pueden analizar y organizar en historias de vida, en autobiografías o historia de vida focal. Estos son formatos que el historiador oral utiliza, de acuerdo al propósito de la investigación $y$ al objeto de estudio, para sistematizar y presentar la evidencia empírica obtenida a través de la técnica de la entrevista. Para algunos autores la historia de vida es una técnica biográfica en la que se recupera el ser y quehacer de una persona en todos los planos de su identidad. Las autobiografías generalmente no son tales, sino que a partir del testimonio directo el historiador logra realizar la biografía de alguien, pero como es realizada con la intervención y visión directa del implicado, por eso se le llama autobiografía. En cambio, la historia de vida focal involucra los relatos de varias personas sobre algún aspecto de su identidad: profesional, personal, familiar, de género, etc. Luego estas historias de vida focalizadas se pueden cruzar y comparar.

La historia de vida no es un método o técnica más, es una perspectiva de análisis única, es capaz de sintetizar lo que la realidad histórica ha hecho de las personas. Un individuo totaliza la sociedad, el sistema se proyecta hacia un individuo; por eso la im- portancia de las mediaciones. El individuo las introyecta horizontal y verticalmente, el individuo es síntesis de los elementos sociales. El sujeto es activo socialmente: se adueña de lo social, lo mediatiza, lo filtra y lo traslada de nuevo proyectándolo en otra dimensión. La cultura está en cada uno de sus miembros y cada uno de sus miembros construye los significados que se pueden leer culturalmente hablando. Las personas no poseen los significados, son poseídas por ellos (Mallimaci y Giménez, 2006).

\section{Reflexiones finales}

Por último, es necesario hacer hincapié de la ética en la producción de la historia oral, lo importante y destacable de la historia oral es que también, es denuncia: puede ser llamada historia moral (Bennett, 1983). El problema está en dilucidar la mirada íntima de la historia oral y la privacidad de la persona.

La historia oral puede entonces convertirse en una importante herramienta de investigación en la educación. Sin circunscribirse solo a su uso dentro de la historiografía educativa, se deberá proponer un uso creativo e innovador de ella, en temáticas como: las identidades de los sujetos que participan en el hecho educativo; la forma en que la estructura social se concretiza en los agentes educativos y a la vez la participación de éstos en la construcción social de la realidad; la posibilidad de utilizarla como portavoz para los actores pocas veces utilizados como informantes (alumnado, mujeres, y responsables de familia ${ }^{\star}$ ), entre muchas otras posibilidades que, quien investiga, pueda encontrar. 
Aunque en la investigación educativa es una opción metodológica y ya se está usando, existen varios puntos que se deben destacar:

En algunas ocasiones se utiliza sin el rigor metodológico necesario para su trabajo. Se argumenta su utilización, sin un sólido enfoque epistemológico que permita dilucidar que los testimonios no son "lo que sucedió", sino lo que la persona entrevistada quiere decir y cómo recuerda lo que aconteció. En pocos estudios educativos donde se ha utilizado la historia oral como método, he percibido esta reflexión y la reflexión desde este plano, en torno al testimonio obtenido.

Por otro lado, aunque no sea el objetivo primordial de este ensayo, se debe ser consciente de la poca sistematicidad de las fuentes orales en educación. En Historia, por ejemplo, existen bancos de datos orales que recuperan los testimonios de participantes en diferentes etapas de la vida política nacional. En educación no existen tales. Por ejemplo, de manera personal he recolectado las entrevistas de los principales actores en una época histórica educativa importante para el estado de Chihuahua: el sexenio panista de Francisco Barrio. Esos testimonios no han sido aprovechados al máximo porque son del dominio particular. Sería muy provechoso, que se intentara crear una fonoteca en la que se abriera un apartado para la recuperación de testimonios orales: la etapa de la educación rural, la implementación del Plan Chihuahua, el sexenio panista, la resistencia del profeso- rado ante los embates educativos neoliberales, etc., son etapas históricas importantes a nivel local que pudiesen ser atesoradas y rescatadas a través de las voces vivas de los participantes. Ya en el futuro se pudiesen realizar el análisis de los mismos.

Otro aspecto que se pudiese impulsar sería la incorporación de investigadores e investigadoras del campo educativo, en redes o agrupaciones de historia oral. La Asociación Internacional de Historia Oral y la Asociación Mexicana de Historia Oral son buenas opciones para relacionarse con personas de otros campos y con mayor experiencia en el trabajo con esta metodología.

La metodología tiene ciertas bondades para la investigación educativa: es económica en los recursos pues no implica el trabajo con muchos informantes, copias, sofisticados software, etc.; sin embargo no es económica en esfuerzo, ni en tiempo. La puede y debe realizar de manera directa el investigador por aquello de que se convierte en el principal instrumento de interpretación. Esto puede significar una ventaja, como también se debe sopesar como desventaja. Depende de lo que se realiza en la investigación.

Por último, tampoco se quiere imponer la visión de la historia oral como «la forma» $\mathrm{o}$ «el camino» en la investigación educativa. Definitivamente, la decisión para tomarla como metodología en el campo, está ligada a la construcción del objeto de investigación realizada por cada investigador. A ellos y ellas dejamos la decisión. 


\section{Notas}

*Se utiliza el término de responsables de familia como una categoría más incluyente que "Padres de familia". Responsables de familia incluye a las madres, las abuelas o abuelos y todas aquellas personas que participan en la educación formal de los educandos.

\section{Referencias}

Aceves, J. (1993). Historia oral. México: Instituto Dr. José María Luis Mora.

Aceves, J. (1996). Caminos y geometría de la historia oral reciente en México. In C. Velasco (Ed.), Historia y testimonios orales (pp. 23-55). México: INAH.

Archila, M. (2005). Voces subalternas e Historia Oral. Anuario Colombiano de Historia Social y de la Cultura/ Universidad Nacional de Colombia, 32, 293308.

Bennett, J. (1983). Human Values in Oral History. The Oral History Review, 11, 1-15.

Bolívar, A., y Domingo, J. (2006). La investigación biográfica y narrativa en Iberoamérica: Campos de desarrollo y estado actual. Forum: Qualitative Social Research, 7(4).

Farías, L., y Montero, M. (2005). De la transcripción y otros aspectos artesanales de la investigación cualitativa. International Journal of Qualitative Methods, 4(1).

Garay, G. d. (1999). La entrevista de historia oral: ¿monólogo o conversación? Revista Electrónica de Investi- gación Educativa, 1(1).

Gaulejac, V. d., y Silva, H. (2002). Memoria e historicidad. Revista Mexicana de Sociología, 64(2), 31-46.

Joutard, P. (1986). Esas voces que nos llegan del pasado. México: FCE.

Mallimaci, F., y Giménez, V. (2006). Historias de vida y método biográfico. In Mallimaci y Giménez (Eds.), Estrategias de Investigación cualitativa. Barcelona: Gedisa.

Morin, E., y Pakman, M. (2003). Introducción al pensamiento complejo. Gedisa Barcelona.

Olivera, M. y. (1971). La historia oral, origen, metodología, desarrollo y perspectivas. Historia Mexicana, 21, No. 2, 372-387.

Portelli, A. (s/f). El tiempo de mi vida. Las funciones del tiempo en la historia oral. In J. Aceves (Ed.), Historia Oral (pp. 195-218). México: Instituto Mora-UAM.

Querzoli, C. P. y. R. (2003). La Entrevista en la Historia de Vida. Algunas Cuestiones Metodológicas. Journal. Retrieved from http://observatoriomemoria.unq. edu.ar/publicaciones/entrevista.pdf

Roncaglia, I. (2004). Analysing Record Interviews: Making Sense of Oral History. Forum: Qualitative Social Research, 5(1).

Thompson, P. (1988). La voz del pasado. Historial oral. Edicions Alfons el Magnanim. Institució Valenciana D’Estudis I Investigació, No 26, 221-261.

Thompson, P. (1993). Historias de vida y análisis del cambio social. In J. Aceves (Ed.), Historia oral (pp. 127-135). México: Instituto Mora/UAM. 